\title{
Resistencia antimicrobiana de Staphylococcus aureus y Streptococcus agalactiae aislados de leche de vacas con mastitis
}

\author{
Quispe, R.; Peña, G.; Andía, V. \\ Laboratorio de Microbiología de Alimentos y Laboratorio de Biología Celular y \\ Molecular, Facultad de Ciencias Biológicas. Universidad Nacional de San \\ Cristóbal de Huamanga, Ayacucho, Perú. E-mail: vidandia(a)yahoo.es
}

\begin{abstract}
Resumen
Quispe, R.; Peña, G.; Andía, V.: Resistencia antimicrobiana de Staphylococcus aureus y Streptococcus agalactiae aislados de leche de vacas con mastitis. Rev. Vet. 32: 1, 79-83, 2021. El uso indiscriminado de los antibióticos sin previa prueba de sensibilidad in vitro es la principal causa del fracaso para el tratamiento de la mastitis en la región de Ayacucho. Con el objetivo de evaluar la resistencia antimicrobiana de Staphylococcus aureus y Streptococcus agalactiae -causantes de la mastitis bovina- se realizó el aislamiento, identificación y el antibiograma de las bacterias en estudio para determinar la resistencia frente a cinco antibióticos. Las muestras fueron recolectadas en el fundo Allpachaca de la Universidad Nacional de San Cristóbal de Huamanga (Ayacucho, Perú), ubicado en las coordenadas $13^{\circ} 23^{\prime} 30.79^{\prime \prime}$ S; $74^{\circ} 15^{\prime} 56.48^{\prime \prime} \mathrm{O}$, con una altitud de $3528 \mathrm{msnm}$. En el campo se evaluaron 94 vacas lecheras utilizando la prueba del California Mastitis Test (CMT) de las cuales resultaron 35 bovinos con mastitis subclínica. En el laboratorio se realizó el aislamiento e identificación de $S$. aureus $(35,2 \%)$ y $S$. agalactiae $(12,7 \%)$ y posteriormente un antibiograma utilizando el método de Baner et al. (1966). Los resultados indicaron que $S$. agalactiae resultó resistente a la penicilina en $22 \%$, a la cefalexina en $22 \%$ y a la sulfatrimetoprima en $33,3 \%$, mientras que para la tetraciclina y para la amoxicilina más ácido clavulánico fueron sensibles al $100 \%$. Por su parte, Staphylococcus aureus resultó resistente a la penicilina en $52 \%$ y la amoxicilina más ácido clavulánico en $28 \%$, mientras que para la cefalexina, tetraciclina y sulfatrimetoprima fueron sensibles al $100 \%$.
\end{abstract}

Palabras clave: mastitis bovina, leche, Staphylococcus aureus, Streptococcus agalactiae, resistencia antimicrobiana.

\begin{abstract}
Quispe, R.; Peña, G.; Andía, V.: Antimicrobial resistance of Staphylococcus aureus and Streptococcus agalactiae isolated from milk from cows with mastitis. Rev. Vet. 32: 1, 79-83, 2021. The indiscriminate use of antibiotics without prior in vitro sensitivity test is the main cause of failure for the treatment of mastitis in the Ayacucho region. In order to assess the antimicrobial resistance of Staphylococcus aureus and Streptococcus agalactiae causing bovine mastitis, the isolation, identification and antibiogram of the bacteria under study were carried out to find the resistance against five antibiotics. Samples were collected at the Allpachaca farm that belongs to the National University of San Cristobal de Huamanga (Ayacucho, Perú), located at coordinates $13^{\circ} 23^{\prime} 30.79^{\prime \prime} \mathrm{S} ; 74^{\circ} 15^{\prime} 56.48$ " $\mathrm{W}$, at an altitude of 3528 meters above sea level. In the field, 94 dairy cows were studied using the California Mastitis Test (CMT), which resulted in 35 cows with subclinical mastitis. In the laboratory, the isolation and the identification of $S$. aureus and $S$. agalactiae were carried out, later the antibiogram was carried out using the method of Bauer et al. (1966). Then $35.2 \%$ of S. aureus and $12.7 \%$ S. agalactiae were isolated. The results of the antibiogram show that S. agalactiae was $22 \%$ resistant to penicillin, $22 \%$ to cephalexin and $33.3 \%$ sulfatrimethoprim, whereas tetracycline, amoxicillin plus clavulanic acid were $100 \%$ sensitive. On the other hand, S. aureus was $52 \%$ resistant to penicillin and amoxicillin plus clavulanic acid $28 \%$, while cephalexin, tetracycline and sulfatrimethoprim were $100 \%$ sensitive.
\end{abstract}

Key words: bovine mastitis, milk, Staphylococcus aureus, Streptococcus agalactiae, antimicrobial resistance. 


\section{INTRODUCCIÓN}

La mastitis bovina es una enfermedad multifactorial que ocasiona pérdidas económicas significativas en la producción de la leche mundial 3,9,20,22,27 . Se caracteriza por la inflamación de la glándula mamaria de las vacas lecheras, causada principalmente por Staphylococcus aureus, S. epidermidis, Streptococcus agalactiae, Escherichia coli y Klebsiella pneumonia, que ocasionan infecciones intramamarias ${ }^{11,15,19}$.

Tales microorganismos existen generalmente en el ambiente donde los animales se encuentran, por tanto, las vacas lecheras son susceptibles a la mastitis, siendo la tasa de infección más alta y frecuente durante la lactancia sucesiva ${ }^{22,29}$.

La mastitis es una compleja y singular enfermedad resultante de la interacción de varios factores asociados con el huésped, bacterias patógenas y el ambiente. La mayoría de los casos de mastitis ocurren en vacas lecheras, por tanto, el tratamiento debe implicar estrategias eficaces e integrales ${ }^{19}$. La terapia antimicrobiana juega un papel determinante en el control de la mastitis al reducir los niveles de infección en el animal y prevenir nuevas infecciones ${ }^{24}$.

El tratamiento de la mastitis clínica se focaliza principalmente en la eliminación de microorganismos infecciosos, y para ello se emplean los antibióticos. No obstante, para seleccionar adecuadamente un antimicrobiano, es necesario conocer el agente etiológico involucrado y la sensibilidad a los antibióticos del medicamento.

Actualmente existen en el mercado diferentes antibióticos para el tratamiento de la mastitis. No obstante, la utilización excesiva de los mismos en el tiempo, está permitiendo la aparición de bacterias patógenos multiresistentes, generando, en algunos casos, el fracaso del tratamiento terapéutico, que puede incluso causar la muerte del animal $18,19,25,26$.

Además, el uso de antibióticos para el tratamiento de la mastitis puede ocasionar residuos en leche constituyendo un problema de salud pública ${ }^{12}$. En la actualidad existe una preocupación mundial por la multi-resistencia y es una de las razones para que algunos países inicien la implementación de programas de monitoreo de resistencia bacteriana y fomenten el uso racional de los antibióticos en animales en producción ${ }^{3,5,18}$.

El uso indiscriminado de los antibióticos sin realizar previamente la prueba de sensibilidad in vitro es la causa principal del fracaso para el tratamiento de la mastitis en algunas regiones del mundo, inclusive en Ayacucho. El inadecuado tratamiento genera por un lado el desarrollo de la resistencia a los antimicrobianos de uso común y, por otro lado, aumenta las pérdidas económicas en la industria láctea.

Por tanto, para garantizar un tratamiento efectivo de la enfermedad, es clave el aislamiento y los estudios de sensibilidad a los antibióticos ${ }^{21,23}$.

\section{MATERIAL Y MÉTODOS}

Desarrollo de la investigación. El trabajo se llevó a cabo en el centro experimental del fundo Allpachaca de la Universidad Nacional de San Cristóbal de Huamanga (UNSCH), Departamento Ayacucho, Perú, ubicado en las coordenadas $13^{\circ} 23^{\prime} 30.79^{\prime \prime} \mathrm{S}$; $74^{\circ} 15^{\prime} 56.48^{\prime \prime}$ O, con una altitud de $3528 \mathrm{msnm}$ y en el Laboratorio de Microbiología Industr. y Aliment. de la Facult. de Cs. Biol. (UNSCH). En Allpachaca se procedió con la prueba CMT en 94 vacas lecheras de la raza Brown Swiss en periodo de ordeño y posteriormente se obtuvieron las muestras de leche de 35 vacas (65 cuartos).

Prueba de California para test de mastitis (CMT). Para la toma de muestras se desecharon los primeros chorros de leche de cada cuarterón y luego ella se colocó en las placas de la paleta, inclinándose la misma para eliminar la mayor parte de la leche obtenida. A la muestra obtenida se le añadió igual volumen de reactivo CMT, se mezcló el reactivo y se examinó el grado de precipitación de acuerdo a la tabla de interpretación de la Prueba CMT ${ }^{9}$

Recolección de muestras de leche para el análisis de laboratorio. La toma de muestras se realizó en el campo durante las primeras horas de la mañana, para ello, antes del ordeño se lavó las ubres, desinfectando con alcohol al $70 \%$. Luego se eliminaron los primeros tres chorros de leche y se colectó $15 \mathrm{ml}$ de leche en un frasco de boca ancha estéril. Los frascos con las muestras se transportaron en una caja de tecnopor a temperatura de $5^{\circ} \pm 2^{\circ} \mathrm{C}$ para su posterior análisis en laboratorio.

Aislamiento e identificación de Staphylococcus aureus y Streptococcus agalactiae. Para realizar el aislamiento se tomó una alícuota de la muestra de leche y se sembró en placas de Petri que contenían agar sangre para $S$. agalactiae para observar el tipo de hemólisis en el cultivo y agar manitol salado para $S$. aureus. Luego se incubaron a $37{ }^{\circ} \mathrm{C}$ por 24 horas. Transcurrido el tiempo, se observaron las características macroscópicas que presentaron las colonias y se realizaron las pruebas: coloración de Gram ${ }^{17}$, prueba de catalasa y coagulasa ${ }^{4}$. También se realizó el test de CAMP para S. agalactiae ${ }^{7}$.

Prueba de resistencia antimicrobiana. Para evaluar dicha prueba de resistencia se utilizó el método de difusión ${ }^{2}$, utilizando los discos de los antibióticos: penicilina, amoxicilina más ácido clavulánico, cefalexina, tetraciclina y sulfatrimetoprima.

Análisis de datos. Los datos obtenidos se procesaron mediante frecuencias, cuadros y gráficos, de acuerdo a los objetivos de la investigación. 


\section{RESULTADOS Y DISCUSIÓN}

Con los resultados obtenidos de 94 vacas (376 cuarterones) de la raza Brown Swiss en el fundo de Allpachaca y utilizando la prueba de CMT (California Mastitis Test), se determinó la frecuencia de $58,1 \%$ para mastitis subclínica, de los cuáles el 16,1\% resultó para reacción traza, $23,9 \%$ para grado $1^{\circ}, 11,4 \%$ para grado $2^{\circ}$ y $6,7 \%$ para grado 3 respectivamente (Figura 1 ).

En Colombia, trabajando con 644 vacas ( 2576 cuarterones) se determinó la producción de leche en base al grado de infección de la mastitis ${ }^{13}$. En Arequipa, de 52 vacas Holstein evaluadas, ocurrió una prevalencia de $40,38 \%$ de mastitis subclínica ${ }^{8}$ (la misma que tiene relación con el estudio realizado), y con respecto a la prueba de CMT reportó $52,73 \%$ para grado $1^{\circ}, 33,21 \%$ para grado $2^{\circ}$ y $14,97 \%$ para grado $3^{\circ}$.

Por otro lado, en la microcuenca Allpachaka (Ayacucho), examinando 347 vacas en su mayoría raza Brown Swiss de 65 rebaños, se determinó la prevalencia de 33,14\% para la microcuenca Allpachaca, 55,13\% para la comunidad Cusibamba, 36,11\% para Manzanayocc, 28,57\% Allpachaca, 26,03\% Satica, 23,33\% Munaypata y $20 \%$ para Unión Paqcha respectivamente ${ }^{16}$.

De los 1388 cuartos mamarios evaluados, el $12,18 \%$ mostraron mastitis subclínica, y con respecto a la prueba de CMT los resultados fueron $28,99 \%$ para reacción traza, $34,32 \%$ para $1^{\circ}$ grado, $33,14 \%$ para $2^{\circ}$ grado y $3,55 \%$ para $3^{\circ}$ grado. Comparando los resultados obtenidos de los autores señalados, no surge ninguna relación con el estudio realizado en el fundo de Allpachaca.

En Ecuador se informó que en la Hacienda San Juan hubo mayor porcentaje de prevalencia de mastitis con el $44,68 \%{ }^{1}$; estos resultados tienen relación con la prevalencia de mastitis sub-clínica encontrados en las vacas con mastitis en el fundo de Allpachaca con un $58,1 \%$.

En la Figura 2 se muestran los resultados de los cuartos afectados según el grado de mastitis en la que se determinó: $24,3 \%$ para el cuarto anterior derecho (CAD), $21,1 \%$ para el cuarto anterior izquierdo (CAI), 27,8\% para el cuarto posterior derecho (CPD) y el $26,8 \%$ para el cuarto posterior izquierdo (CPI).

De un total de 52 vacas evaluadas, el cuarto con mayor prevalencia fue el cuarto posterior izquierdo (CPI) con $33,3 \% \mathrm{y}$ el cuarto anterior izquierdo (CAI) con $30 \%$, resultados obtenidos que son similares al presente estudio ${ }^{8}$.

Los resultados obtenidos en el aislamiento de $S$. agalactiae y $S$. aureus aislados chaca. pachaca. de leche de vacas con mastitis del fundo Allpachaca fueron 12,7\% para Streptococcus agalactiae, 35,2\% para Staphylococcus aureus, $16,2 \%$ para Streptococcus $s p, 21,1 \%$ para Staphylococcus $s p$ y $14,1 \%$ no mostró crecimiento (Figura 3).

Los resultados obtenidos en el fundo de Allpachaca tienen relación con un estudio realizado en la Sabana de Bogotá (Colombia) ${ }^{13}$, que encontró mayor prevalencia a Staphylococcus aureus, seguido de Streptococcus agalactiae.

Por otro lado, se determinó 73,28\% para Staphylococcus aureus y $8,40 \%$ para Streptococcus agalactiae;

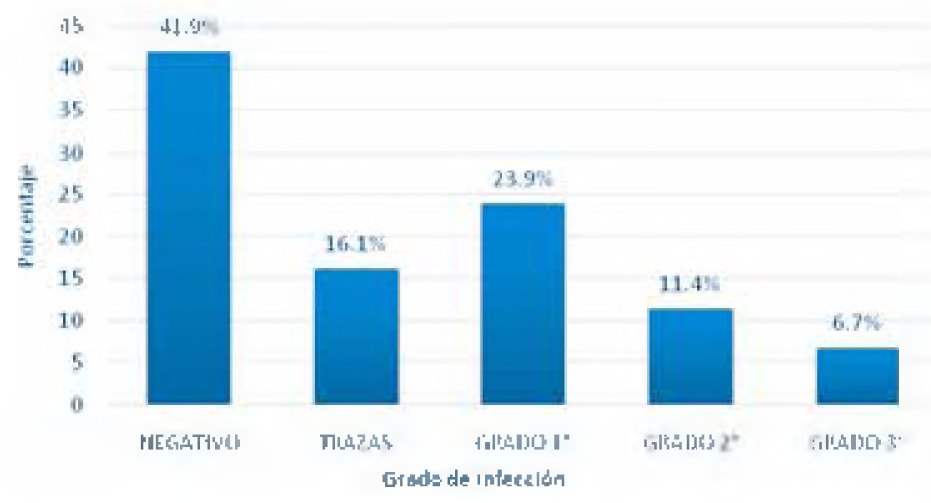

Figura 1. Frecuencia de mastitis según el grado de infección por la prueba de CMT realizada en vacas lecheras del fundo Allpa-

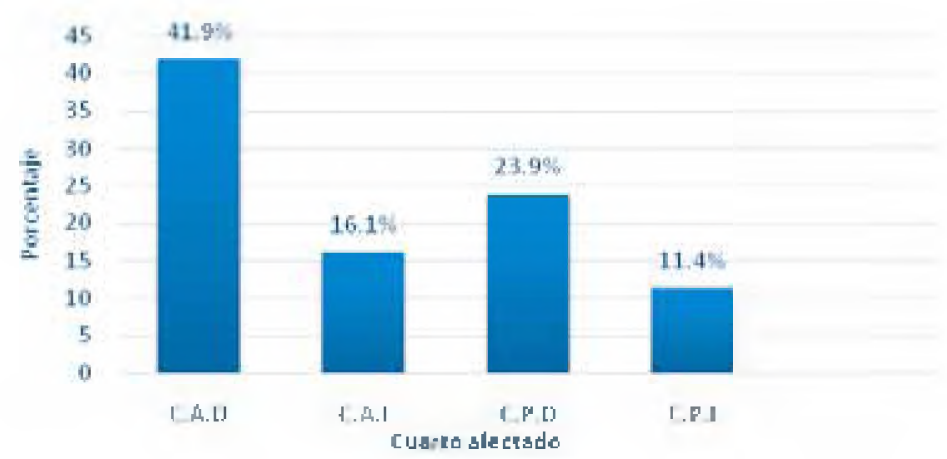

Figura 2. Frecuencia de cuartos afectados según grado de mastitis tomadas a partir de trazas: gradoa $1^{\circ}, 2^{\circ}$ y $3^{\circ}$ en vacas lecheras de Allpachaca.

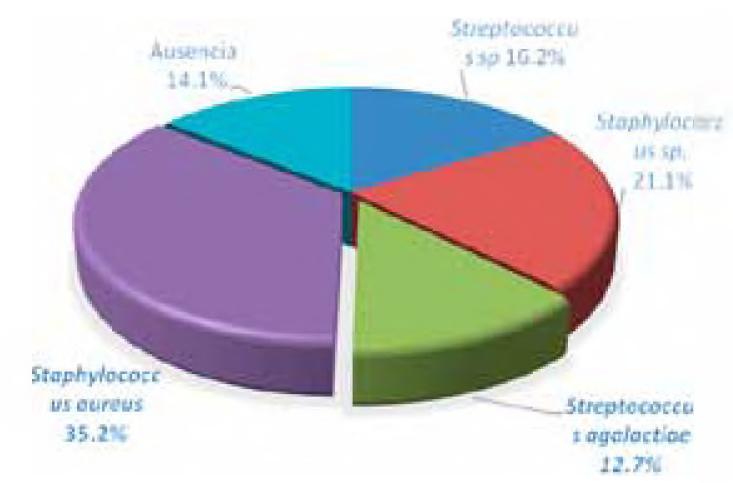

Figura 3. Frecuencias de Staphylococcus aureus y Streptococcus agalactiae aisladas de leche de vacas con mastitis del fundo All- 


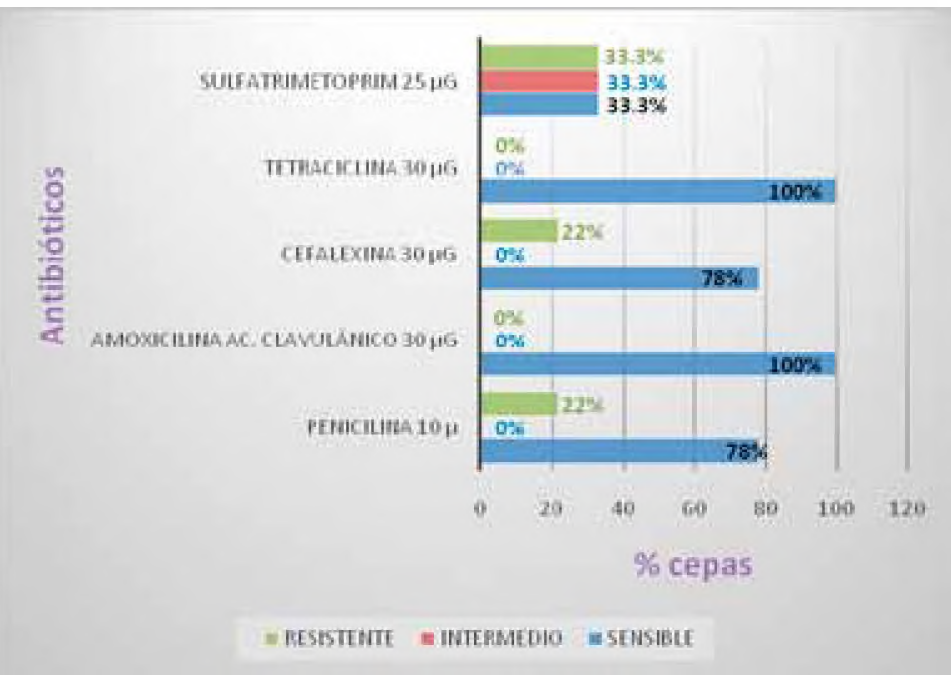

Figura 4. Resistencia antimicrobiana de Streptococcus agalactiae aislado de leche de vacas con mastitis del fundo Allpachaca.

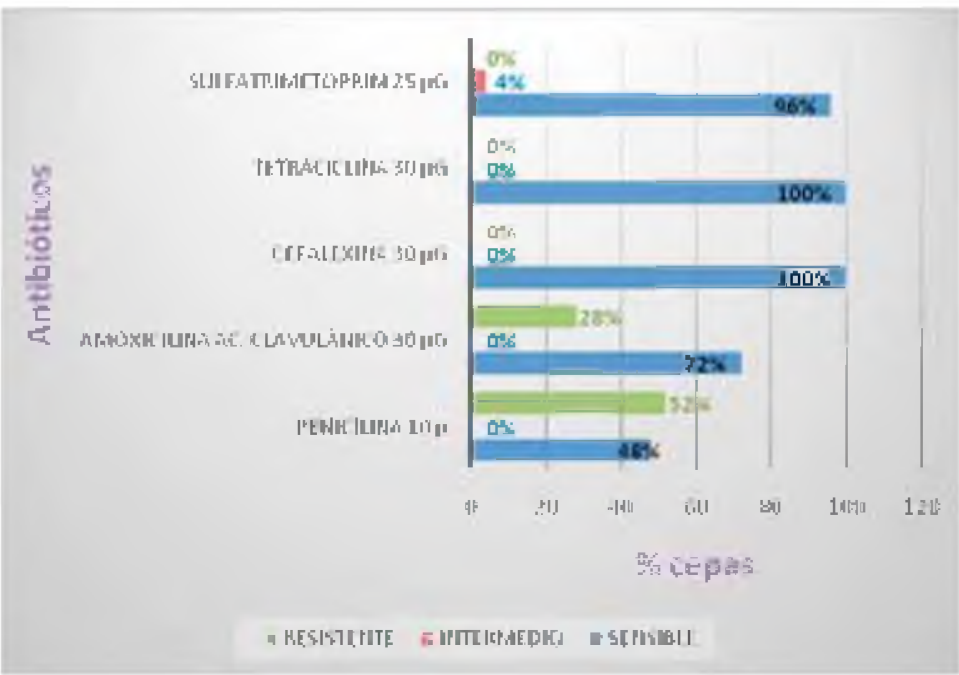

Figura 5.Resistencia antimicrobiana de Staphylococcus aureus aislada de leche de vacas con mastitis del fundo Allpachaca.

también se reportaron $16,34 \%$ para Streptococcus $s p$ y $34,64 \%$ para Staphylococcus sp ${ }^{16}$. Ambos trabajos señalados tienen similitud con los resultados del presente trabajo.

Los resultados del antibiograma indican que Streptococcus agalactiae resultó resistente a la penicilina en $22 \%$, a cefalexina en $22 \%$ y sulfatrimetoprima en $33,3 \%$, mientras que para la tetraciclina como para la amoxicilina más ácido Clavulánico, fueron sensibles al $100 \%$ (Figura 4).

Por otro lado, Staphylococcus aureus resultó resistente a la penicilina en $52 \%$ y la amoxicilina más ácido clavulánico en $28 \%$, mientras que para la cefalexina, tetraciclina y sulfatrimetoprim fueron sensibles al $100 \%$ (Figura 5).

En vacas con mastitis resistentes a tetraciclina $(72,5 \%)$, se determinaron: gentamicina $(29,4 \%)$, amikacina $(29,4 \%)$ y eritromicina $(23,5 \%){ }^{10}$. Los agentes antimicrobianos disponibles actualmente para tratar la mastitis bovina, demostraron una buena actividad contra las cepas de $S$. aureus. Además, indican que penicilina + novobiocina demostró una actividad comparable a la amoxicilina + clavulanato contra Staphylococcus aureus ${ }^{6}$.

Por otro lado, Staphylococcus aureus es el patógeno etiológico más común de la mastitis bovina. Las cepas resistentes hacen que la enfermedad sea difícil de tratar la mastitis ${ }^{28}$. Además, en casos de mastitis bovina en el noroeste de China se señaló que $S$. aureus es resistente a la penicilina $(84,09 \%)$, eritromicina $(20,45 \%)$, tetraciclina $(15,91 \%)$, gentamicina $(9,09 \%)$, tobramicina $(6,82 \%)$, kanamicina $(6,82 \%)$ y meticilina $(2,27 \%)$.

El $9,09 \%$ de $S$. aureus fue multirresistente. Además, reportó resistencia a rifampicina $(100 \%)$, penicilina $(95,45 \%)$, tetraciclina $(22,73 \%)$, eritromicina $(22,73 \%)$ y vancomicina $(2,27 \%)$.

Se demostró la susceptibilidad a diversos antibióticos, siendo Staphylococcus aureus susceptibles a ceftriaxona $80,88 \%$, cefotaxima $79,41 \%$, ciprofloxacin $73,52 \%$, eritromicina $70,05 \%$, amikacina $69,11 \%$, gentamicina $52,94 \%$, amoxicilina $36,76 \%$, ampicilina $29,41 \%$ y la menor susceptibilidad se mostró con la penicilina: $13,23 \%$.

La prueba de susceptibilidad antibacteriana de Staphylococcus aureus a varios antibióticos reveló que el $86,76 \%$ eran resistentes a la penicilina seguidos de ampicilina $70,50 \%$, amoxicilina $63,23 \%$, gentamicina $47,05 \%$, amikacina $30,80 \%$, eritromicina $27,94 \%$, ciprofloxacina $26,47 \%$, meticilina $23,52 \%$, cefotaxima $20,58 \%$ y la menor resistencia se mostró en ceftriaxona: $19,11 \%$.

Asimismo, se mencionó la existencia de un alarmante nivel de resistencia de las bacterias de la mastitis a los agentes antimicrobianos. Por lo tanto, resulta necesaria una prueba de susceptibilidad antes del uso de antibióticos, tanto en el tratamiento como en la prevención de las infecciones intramamarias ${ }^{24}$.

No obstante, se deben realizar exámenes para la detección de mastitis subclínica y también para el tratamiento adecuado del cuadro clínico de vacas durante el período de lactancia ${ }^{29}$. La alta frecuencia de genes de virulencia y resistencia genética en los aislamientos, constituyen una razón principal para el fracaso del tratamiento y posiblemente conducen a la propagación de la resistencia ${ }^{14}$.

Agradecimiento: al Médico Veterinario Diomedes Ataucusi Yupanqui, Administrador de la Unidad de Producción de Bienes de Allpachaca, UNSCH. 


\section{REFERENCIAS}

1. Acuña V, Rivadeneira EP. 2008. Aislamiento, identificación y antibiograma de patógenos presentes en leche con mastitis en ganaderías bovinas de la Provincia de Pichincha. Ecuador. Tesis de grado. Escuela Politécnica del Ejército, Pichincha. Ecuador, p. 90-115.

2. Bauer AW, Kirby WM, Sherris JC. 1966. Antibiotic susceptibility testing by a standardized single disk method. Am J Clin Pathol 45: 493-496.

3. Bedolla C, Deleón M. 2008. Pérdidas económicas ocasionadas por la mastitis bovina en la industria lechera. Red Vet.9: 1-26.

4. Bennett RW, Lancette GA. 1995. Bacteriological analytical manual, $8^{\mathrm{a}}$ ed., AOAC Internat., Galthersburg, 355 p.

5. Bhosale RR et al. 2014. Mastitis: an intensive crisis in veterinary science. International Journal of Pharma Research and Health Sciences, 2: 96-103.

6. Deoliveira AP. 2000. Antimicrobial susceptibility of Staphylococcus aureus isolated from bovine mastitis in Europe and the United States. J Dairy Sci 83: 855-862.

7. Denamiel G, Puigdevall T, Chan D, Gentilini E. 2013. Mastitis bovina: variaciones en la detección de $\beta$-hemolisina y factor CAMP para la identificación de Streptococcus agalactiae. Rev Med Vet (Buenos Aires) 94: 24-27.

8. Dueñas B. 2007. Prevalencia de mastitis subclínica bovina y determinación de sólidos totales de la leche en la Irrigación Majes sección (B2), Arequipa. Tesis de pregrado, Univ Nac Antipl Puno (Perú), p. 38-47.

9. Figueroa P, Bedolla J. 2008. Determinación de la prevalencia de mastitis bovina en el municipio de Tarímbaro, Michoacán, mediante la prueba de California. Rev Electr Vet 9: 10 .

10. Gao JF et al. 2012. Antibiotic resistance of Streptococcus agalactiae from cows with mastitis. The Vet Journal 194: 423-424.

11. Godden SM et al. 2002. The effect of sampling time and sample handling on the detection of Staphylococcus aureus in milk from quarters with subclinical mastitis. The Canad Vet J 43: 38.

12. Leal M. 2014. Antibacterial efficacy of plant extracts: clinical application in bovine mastitis. Rev UDCA Actualidad \& Divulgación Cientifica 17: 179-187.

13. Martínez GR. 2006. Comportamiento de la mastitis bovina y su impacto económico en algunos hatos de la Sabana de Bogotá, Colombia. Revista Medicina Veterinaria, 12 : 35-55.

14. Memon J et al. 2013. Genotypes, virulence factors and antimicrobial resistance genes of Staphylococcus aureus isolated in bovine subclinical mastitis from eastern China. Pakistan Veterinary Journal 33: 4.
15. Oliveira L, Hulland C, Ruegg P. 2013. Characterization of clinical mastitis occurring in cows on 50 large dairy herds in Wisconsin. J Dairy Science 96: 7538-7549.

16. Paqui, E. 2011. Prevalencia e identificación de agentes bacterianos causales de mastitis subclínica bovina en la microcuenca Allpachaka, Ayacucho. 2008. Tesis, Univ. Nac. San Crist. de Huamanga, Ayacucho, Perú, p. 37-57.

17. Perilla MJ et al. 2009. Manual de laboratorio para la identificación y prueba de susceptibilidad a los antimicrobianos de patógenos bacterianos de importancia para la salud pública en el mundo en desarrollo. Medicina \& Laboratorio, 15: 69-91.

18. Sanmartín B et al. 2002. Resistencia bacteriana en cepas patógenas aisladas de mastitis en vacas lecheras de la $\mathrm{V}$ Región, Región Metropolitana y X Región, Chile. Arch Med Vet 34: 2, 221-234.

19. Sharif A, Umer M, Muhammad G. 2009. Mastitis control in dairy production. Journal Agricult \& Social Sci 5: 102-105.

20. Souza FN et al. 2016. Somatic cell count and mastitis pathogen detection in composite and single or duplicate quarter milk samples. Pesq Vet Brasileira, 36: 811-818.

21. Sumathi BR, Veeregowda BM, Amitha RG. 2008. Prevalence and antibiogram profile of bacterial isolates from clinical bovine mastitis. Vet World 8: 237-238.

22. Tineo J, Andía V. 2017. Mastitis bovina por recuento de células somáticas con Porta SCC y Test de California en el fundo de Allpachaca-UNSCH, Ayacucho, Perú. Rev Electr Vet (REDVET) 18: 7

23. Turnidge J, Paterson D. 2007. Setting and revising antibacterial susceptibility breakpoints. Clin Microbiol Reviews 20: 391-408.

24. Unakal C, Kaliwal B. 2010. Prevalence and antibiotic susceptibility of Staphylococcus aureus from bovine mastitis. Vet World 3: 65.

25. Valenzuela M. 2010. Sensibilidad antimicrobiana de Staphylococcus aureus aislados de mastitis clínica bovina en rebaños lecheros de la Región de Los Ríos. Tesis de grado, Universidad Austral de Chile, p. 25-31.

26. World Health Organization (WHO). 2000. Overcoming antimicrobial resistance, Geneva, Switzerland, $67 \mathrm{p}$.

27. Wolter W, Castañeda H, Kloppert B, Zschöck M. 2004. Mastitis bovina. Prevención, diagnóstico y tratamiento. Ed. Universit., Guadalajara, Jalisco, 16: 62-72.

28. Yang F et al. 2016. Genetic characterization of antimicrobial resistance in Staphylococcus aureus isolated from bovine mastitis cases in Northwest China. Journal of Integrat Agricult 15: 2842-2847.

29. Zeryehun T, Abera G. 2017. Prevalence and bacterial isolates of mastitis in dairy farms in selected districts of eastern Harrarghe Zone, Eastern Ethiopia. Journal of Vet Med 17: 2-7. 